\title{
High-speed Dynamic Lab-based Micro-CT for Optically Opaque Flows
}

Jan Dewanckele ${ }^{1}$, Marijn Boone ${ }^{1}$, Luke Hunter ${ }^{2}$, Andreas Griesser ${ }^{3}$, Christian Wagner ${ }^{3}$ and Simo Makiharju $^{4}$

${ }^{1}$ TESCAN XRE, Gent, Oost-Vlaanderen, Belgium, ${ }^{2}$ TESCAN USA, Warrendale, Pennsylvania, United States, ${ }^{3}$ Math2Market, Kaiserslautern, Rheinland-Pfalz, Germany, ${ }^{4}$ University of California, Berkeley, Berkeley, California, United States

Single and multi-phase flow are encountered almost everywhere, including biological and chemical processing, offshore applications, oil and gas recovery, corrosion and naval hydrodynamics. Extending the physical understanding of single and multi-phase flow is of key importance of many industrial applications.

High-speed visualization, such as particle image velocimetry (PIV) has been successfully applied to capture flow processes in detail for many of these flows. However, for some specific set-up, light might scatter between vapor and liquid phases or more severely, be completely blocked. To overcome this limitation of PIV, a medical X-ray source coupled with a high-speed imager was used to study the void fractions and two-dimensional wedges in ventilated and cavitating flows (Mäkiharju et al., 2013). The system was capable of acquiring two-dimensional projections through $O(10 \mathrm{~cm})$ sized water column at rates in the order of $1 \mathrm{kHz}$ with spatial resolution below $1 \mathrm{~mm}$.

In general, when using experimental techniques to study the behavior of flow, they ideally should have both a high spatial and temporal resolution to resolve the dynamics of the flows. Because of their high fluxes, synchrotron facilities are well suited to perform those dynamic experiments (Duke et al.,2015). Time-resolved 3D imaging with X-rays has rapidly emerged as an essential technique to understand materials evolution, facilitating in situ investigations ranging from mechanical deformation to fluid flow in porous materials and beyond. Imaging of dynamic processes is one of the key applications at synchrotron facilities, pushing the time resolution more and more down with quite some success. However, access to those facilities is often limited and operational cost are quite high.

The flows of industrial interest requires typically imaging rates much higher than achievable with conventional single micro-CT laboratory systems. If the acquisition time is slower that the voxel size over flow speed, temporal blurring is induced. However, recent developments at TESCAN XRE have made it possible to image, reconstruct and inspect dynamic processes in the laboratory with a temporal resolution below 10 seconds, meaning that an entire acquisition from 0 to $360^{\circ}$ is completed within 10 seconds. These developments are enabling investigation of low Reynolds number opaque flows in great detail. We study these flows also to advance the X-ray techniques that are equally applicable to higher speed flows. Moreover, with the constant development of faster scan acquisitions, the practical implementations of these routines are expected to vastly increase.

The present investigation exploits the use of high-speed laboratory based micro-CT cone beam system to visualize and quantify three dimensional flows. Silver coated particles $O$ (40 $\mu \mathrm{m}$ diameter) were used as flow tracers in a liquid (glycerol). An isotropic voxel size of $15 \mu \mathrm{m}$ was obtained for the scans and has been considered adequate to image the silver coated particles. Figure 1 shows three snapshots of an uninterrupted dynamic experiment of 10 minutes. Hundred full rotation were acquired with a total scan 
time per rotation $\left(0-360^{\circ}\right)$ of 5.8 seconds. The bright spots are the silver coated particles and the red arrow indicated a relatively faster moving $(\sim 10 \mu \mathrm{m} / \mathrm{s})$ air void in the liquid.

The study demonstrated the ability on a laboratory scale system to provide Lagrangian particle trajectories, thus capturing in three dimensions the effects of changing flow parameters.

\section{ACKNOWLEDGEMENTS}

The last author gratefully acknowledges the support of NSF EAGER award \#1922877 program manager Ron Joslin and Shahab Shojaei-Zadeh.

References

Duke, D., Swantek, A., Kastengren, A., Fezzaa, K., \& Powell, C. (2015). Recent Developments in X-ray Diagnostics for Cavitation. SAE International Journal of Fuels and Lubricants, 8(1), 135-146. https://doi.org/10.4271/2015-01-0918

Mäkiharju, S. A., Gabillet, C., Paik, B. G., Chang, N. A., Perlin, M., \& Ceccio, S. L. (2013). Time-resolved two-dimensional X-ray densitometry of a two-phase flow downstream of a ventilated cavity. Experiments in Fluids, 54(7), 1-21. https://doi.org/10.1007/s00348-013-1561-z 